\title{
Dynamic measurement of the optical properties of bovine enamel demineralization models using four- dimensional optical coherence tomography
}

\author{
Abdirahman Aden \\ Arthi Anthony \\ Carel Brigi \\ Muhammad Sabih Merchant \\ Huda Siraj \\ Peter H. Tomlins
}




\title{
Dynamic measurement of the optical properties of bovine enamel demineralization models using four-dimensional optical coherence tomography
}

\author{
Abdirahman Aden, Arthi Anthony, Carel Brigi, Muhammad Sabih Merchant, Huda Siraj, and Peter H. Tomlins* \\ Queen Mary University of London, Institute of Dentistry, Barts and The London School of Medicine and Dentistry, London, United Kingdom
}

\begin{abstract}
Dental enamel mineral loss is multifactorial and is consequently explored using a variety of in vitro models. Important factors include the presence of acidic $\mathrm{pH}$ and its specific ionic composition, which can both influence lesion characteristics. Optical coherence tomography (OCT) has been demonstrated as a promising tool for studying dental enamel demineralization. However, OCT-based characterization and comparison of demineralization model dynamics are challenging without a consistent experimental environment. Therefore, an automated four-dimensional OCT system was integrated with a multispecimen flow cell to measure and compare the optical properties of subsurface enamel demineralization in different models. This configuration was entirely automated, thus mitigating any need to disturb the specimens and ensuring spatial registration of OCT image volumes at multiple time points. Twelve bovine enamel disks were divided equally among three model groups. The model demineralization solutions were citric acid $(\mathrm{pH} 3.8)$, acetic acid $(\mathrm{pH} 4.0)$, and acetic acid with added calcium and phosphate ( $\mathrm{pH} 4.4)$. Bovine specimens were exposed to the solution continuously for $48 \mathrm{~h}$. Three-dimensional OCT data were obtained automatically from each specimen at a minimum of 1-h intervals from the same location within each specimen. Lesion dynamics were measured in terms of the depth below the surface to which the lesion extended and the attenuation coefficient. The net loss of surface enamel was also measured for comparison. Similarities between the dynamics of each model were observed, although there were also distinct characteristic differences. Notably, the attenuation coefficients showed a systematic offset and temporal shift with respect to the different models. Furthermore, the lesion depth curves displayed a discontinuous increase several hours after the initial acid challenge. This work demonstrated the capability of OCT to distinguish between different enamel demineralization models by making dynamic quantitative measurements of lesion properties. This has important implications for future applications in clinical dentistry. () The Authors. Published by SPIE under a Creative Commons Attribution 3.0 Unported License. Distribution or reproduction of this work in whole or in part requires full attribution of the original publication, including its DOI. [DOI: 10.1117/1.JBO.22.7.076020]
\end{abstract}

Keywords: demineralization; optical coherence tomography; lesion progression.

Paper 160795RR received Nov. 17, 2016; accepted for publication Jul. 13, 2017; published online Jul. 31, 2017.

\section{Introduction}

Dental hard tissue disease is frequently characterized by mineral loss. Hence, this is a critical factor in its diagnosis and management in terms of carious and erosive lesions. Clinical diagnosis is based upon anatomical site, recurrence, disease progression, extent, tissue involvement, and chronology. ${ }^{1,2}$ Both acid erosion and caries are driven by acidic $\mathrm{pH}$, either due to external agents (e.g., citrus juices) or naturally occurring metabolites such as lactic acid. Consequently, the two conditions are not necessarily mutually exclusive. ${ }^{3}$ However, lesion characteristics are not governed by $\mathrm{pH}$ alone, exhibiting dependence on the composition of the demineralizing agent. ${ }^{4}$ Simplified in vitro models have been developed to study dissolution chemistry, etiological factors, and symptomatic features. ${ }^{4}$ Furthermore, several analytical techniques have found utility for measuring dental mineralization. Transverse microradiography has been used to assess lesion depth and mineral density, ${ }^{5}$ along with microhardness ${ }^{6,7}$ testing and scanning electron microscopy for evaluating structural changes that occur during demineralization. ${ }^{8} \mathrm{X}$-ray

*Address all correspondence to: Peter H. Tomlins, E-mail: p.h.tomlins@qmul. ac.uk microtomography has provided quantitative high-resolution three-dimensional (3-D) mapping of dental lesions ${ }^{9}$ and mineral density. ${ }^{10}$ These laboratory techniques have contributed to both the understanding of dental lesions and the development of oral healthcare treatments. However, they are not readily translated directly into the clinic for in vivo patient examination. Consequently, alternative measurement and imaging technologies have been reported in the dental research literature. These include quantitative light fluorescence and optical coherence tomography (OCT), ${ }^{11}$ which can be used for both laboratory-based in vitro experiments and in vivo clinical research. OCT is attractive because it noninvasively images backscattered light intensity, which has been shown to be sensitive to changes in dental demineralization. ${ }^{12-16}$

OCT measurements of lesions formed under different demineralizing conditions have not previously been compared using a consistent environment. Furthermore, OCT studies have previously been limited by protocols that require specimens to be moved between demineralization solutions and the imaging equipment. This is a major limitation for longitudinal studies that aim to understand lesion dynamics over time because measurement uncertainty is associated with specimen realignment at each imaging step. ${ }^{17}$ 
Therefore, this paper reports a "hands-free" experimental configuration that mitigates against the need for specimen movement at each time point. The primary aim of developing this system was to enable longitudinal OCT measurements of dental mineralization. This paper investigated whether previously established OCT-based measurements could distinguish between lesions formed using different demineralization models.

\section{Theory}

The OCT image forming signal is the result of optical backscatter from within a specimen. Spatial variations in the scattering properties of materials lead to image contrast, enabling regions with different optical properties, such as different tissue types, ${ }^{18}$ to be visualized. Furthermore, disease processes have been shown to modify the scattering properties of biological tissue. ${ }^{19-22}$ In the single scattering regime, the OCT signal $(I)$ can be approximated by an exponential decay of the form given as ${ }^{3-25}$

$I(z)=\mu_{\mathrm{B}} \exp \left[-2 \mu_{\mathrm{OCT}}\left(z-z_{0}\right)\right]$,

where $z_{0}$ represents the axial starting point of a region described by homogeneous optical properties. The optical properties are expressed in terms of the backscattering coefficient $\mu_{\mathrm{B}}$ and the signal attenuation constant $\mu_{\mathrm{OCT}}$. The signal attenuation constant describes the rate of OCT intensity decrease due to both absorption and scattering. It is a linear combination of the absorption coefficient $\mu_{\mathrm{a}}$ and the scattering coefficient $\mu_{\mathrm{s}}$ such that $\mu_{\mathrm{OCT}}=\mu_{\mathrm{a}}+\mu_{\mathrm{s}}$.

The phase function is a probability distribution that statistically relates the intensity of light from an incident direction that couples via single scattering into a given scattered direction. The phase function, $p(\theta)$, can be summarized by the average cosine of the scattering angle, $\theta$. This is called the anisotropy factor, $g$, where $g=0$ is equivalent to isotropic scattering, $g=$ -1 total backscatter, and $g=1$ total forward scatter.

Both $\mu_{\mathrm{B}}$ and $\mu_{\mathrm{s}}$ are proportional to the phase function ${ }^{23,26}$ integrated over scattering angles $(0$ to $\pi)$ and $[\pi$-arctan numerical aperture (NA) to $\pi$ ], respectively. Furthermore, the ratio $\mu_{\mathrm{B}} / \mu_{\mathrm{s}}=p_{\mathrm{NA}}$ is the probability that light will be scattered into the NA of the OCT objective lens. Therefore, if the shape of the phase function is known, $g$ can also be determined. ${ }^{26,27}$

The dominance of either scattering or absorption is described by the albedo, ${ }^{28} a=\mu_{\mathrm{s}} /\left(\mu_{\mathrm{a}}+\mu_{\mathrm{s}}\right)$ such that $a=0$ indicates pure absorption and $a=1$ indicates purely scattering. In applications such as ophthalmology, the operating waveband of OCT can be chosen to minimize optical absorption, ${ }^{29}$ leading to the assumptions that $a \approx 1$ and, therefore, $\mu_{\mathrm{OCT}} \approx \mu_{\mathrm{s}}$ while maintaining a useful imaging depth. ${ }^{30}$ Healthy dental enamel is reported to comprise $\sim 87 \%$ to $93 \%$ mineral by volume. ${ }^{31}$ Hydrated enamel has been reported to exhibit an increased OCT imaging depth compared to dried enamel, ${ }^{32}$ most likely by refractive index matching in the interprismatic region. Furthermore, at an optical wavelength of $1300 \mathrm{~nm}$, water absorption is much smaller than scattering from enamel. ${ }^{33,34}$ Therefore, it is assumed that in dental enamel scattering remains the dominant source of signal attenuation.

In real OCT systems, the measured OCT signal, $I_{\mathrm{m}}$, is the product of $I(z)$ with the axial coupling efficiency $(T)$ centered around the focus at $z_{f}$ and the axial sensitivity roll-off $(H)$

$I_{\mathrm{m}}(z)=\alpha T\left(z-z_{f}\right) H(z) I(z)$.

The calibration scale factor, $\alpha$, facilitates a comparison between different specimens and systems by accounting for systematic coupling efficiency differences. Calibration of the axial variations in detection sensitivity and optical coupling efficiency is necessary when comparing measurements of the optical properties obtained from different axial positions. Typically, $\mu_{\mathrm{OCT}}$ and $\mu_{\mathrm{B}}$ are obtained from a least squares fit of Eq. (1) to segments of OCT A-scans following calibration of the term $\alpha T\left(z-z_{f}\right) H(z)$. Calibration can be achieved by measuring a specimen with known optical properties. ${ }^{23,24}$ However, even if the anisotropy factor is unknown, a homogeneous scattering phantom with known attenuation coefficient $\mu_{\text {cal }}$ can still provide a relative intensity calibration. Dividing the OCT A-scan profile of a specimen $I_{\mathrm{m}}$ by an equivalent calibration, A-scan yields the expression for the corrected signal

$I_{\text {corr }}(z)=\mu_{\mathrm{B}}^{\prime} \exp \left[-2 \mu_{\mathrm{OCT}}^{\prime}\left(z-z_{0}\right)\right]$

where $\mu_{\mathrm{B}}^{\prime}=\mu_{\mathrm{B}}(g) / \mu_{\mathrm{B}}\left(g_{\mathrm{cal}}\right)$ and $\mu_{\mathrm{OCT}}^{\prime}=\mu_{\mathrm{OCT}}-\mu_{\mathrm{cal}}$. The subscript "cal" denotes the properties of the calibration phantom. Therefore, $\mu_{\mathrm{OCT}}^{\prime}$ can be obtained experimentally by fitting Eq. (3) to a corrected OCT A-scan. The specimen attenuation coefficient is found from the sum $\mu_{\mathrm{OCT}}=\mu_{\mathrm{OCT}}^{\prime}+\mu_{\text {cal }}$.

These expressions have facilitated the use of OCT for quantitative evaluation of the optical properties of collagen gel tissue scaffolds ${ }^{35}$ and biological tissues, ${ }^{36}$ such as atherosclerotic plaques, ${ }^{25,37,38}$ epithelial precancer, ${ }^{39,40}$ dental caries, ${ }^{11,14,41,42}$ and enamel structure. ${ }^{43}$ These methods have been extended to the spatial mapping of $\mu_{\mathrm{OCT}}$, a technique termed both parametric $\mathrm{OCT}^{44}$ and scattering attenuation microscopy. ${ }^{45}$

In general, it is preferred to measure the calibration function, rather than rely upon a theoretical description. However, in the presence of dental erosion, the specimen surface moves with respect to the system objective lens. Refractive index differences between the specimen and its surrounding medium necessarily lead to a shift in the focal plane position, $z_{f}$. Geometrical optics can be used to estimate the expected focal plane shift due to erosion using the following expression: ${ }^{46}$

$\Delta z_{f}=\frac{r_{1}+\tan \theta_{1}\left(z_{0}-z_{\text {lens }}\right)}{\tan \theta_{2}}+z_{0}-z_{f}^{\prime}$.

The angle, $\theta_{1}$, denotes the peripheral ray of a focused beam propagating through a medium having refractive index $n_{1}$, where the focusing lens is located at $z_{\text {lens }}$ along the optical axis, at which the collimated beam radius is $r_{1}$. A specimen having refractive index $n_{2}$ is located such that its surface is perpendicular to the optical axis at $z_{0}$. Upon passing into the second medium, the peripheral ray is refracted to a new angle, $\theta_{2}=\sin ^{-1}\left\{\left[n_{1} \mathrm{NA}\right] / n_{2}\right\}$. The axial location at which the beam would have come to focus in the absence of the specimen is $z_{f}^{\prime}$.

Assuming an objective NA of $\mathrm{NA}=\sin \theta_{1}=0.05, r_{1}=$ $1.5 \mathrm{~mm}, z_{\text {lens }}=0 \mathrm{~mm}, z_{0}=2 \mathrm{~mm}, n_{1}=1.32$ (water), ${ }^{47,48} n_{2}=$ 1.63 (enamel), ${ }^{49}$ and $z_{f}^{\prime}=30 \mathrm{~mm}$, then a $100-\mu \mathrm{m}$ shift in the surface position, $z_{0}$, corresponds with a focal shift of $124 \mu \mathrm{m}$.

The coupling efficiency term, $T$, in Eq. (2) is sensitive to the focal position according to the following equation:

$T\left(z-z_{\mathrm{f}}\right)=\frac{1}{\left[\frac{\left(z-z_{\mathrm{f}}\right) \lambda_{0}}{2 n_{2} \pi w_{0}^{2}}\right]^{2}+1}$,

where $w_{0}$ is the beam waist at the focus $\left(z_{f}\right)$ with illumination wavelength of $\lambda_{0}=1300 \mathrm{~nm}$. 
The measured OCT intensity is a product of the coupling efficiency and the scattering slope. It, therefore, follows that variation in the focal plane will lead to variation in measurements of $\mu_{\mathrm{OCT}}$. This uncertainty was explored by a Monte Carlo simulation whereby a uniform distribution of 1000 focal shifts was generated over the range $\pm 200 \mu \mathrm{m}$, and corresponding OCT signals were produced using Eq. (2) with $\mu_{\mathrm{OCT}}=1 \mathrm{~mm}^{-1}$ and $H=1$. An exponential function of the form given in Eq. (3) was fitted to each of the simulated OCT curves, resulting in a distribution of estimates for $\mu_{\mathrm{OCT}}$. The standard deviation was equivalent to a $5 \%$ variation from the expected value.

\section{Materials and Methods}

\subsection{Bovine Enamel Specimens}

Twelve bovine incisors were prepared and embedded into 25 mm-diameter clear resin substrates. Each tooth was sectioned into a 6-mm-diameter disk, sliced in the coronal plane to an approximate thickness of $2.6 \mathrm{~mm}$. The enamel surface was exposed by polishing to a flat specular finish, leaving a residual enamel thickness of $\sim 0.5 \mathrm{~mm}$ on top of the underlying dentine. The specimens were prepared by Modus Laboratories Ltd., Reading, UK.

\subsection{Artificial Lesion Models}

Artificial lesions were produced by continuous exposure of the enamel to one of three different demineralization solutions.

\subsubsection{Citric acid "erosion model" ( $\mathrm{pH}$ 3.8)}

A diluted citric acid model, previously used for erosion studies, ${ }^{50-52}$ was prepared as $0.05 \mathrm{M}$ citric acid (Sigma-Aldrich, $\mathrm{UK}$ ). The solution was buffered to $\mathrm{pH} 3.8$ using sodium hydroxide $(\mathrm{NaOH})$ pellets.

\subsubsection{Acetic acid "caries-like demineralization" model (pH 4.0)}

A diluted acetic acid model, previously reported for demineralization studies, ${ }^{53}$ was prepared as $0.17 \mathrm{M}$ acetic acid, buffered to $\mathrm{pH} 4.0$ by the addition of $\mathrm{NaOH}$ pellets (Sigma-Aldrich, UK).

\subsubsection{Acetic acid "caries-like" model ( $\mathrm{pH}$ 4.4)}

Caries models typically contain a source of calcium and phosphate, enabling a subsurface lesion to form while maintaining an intact surface. ${ }^{54-57}$ Thus, the caries-like model solution was prepared as $2.2 \mathrm{mM} \mathrm{CaCl}, 2.2 \mathrm{mM} \mathrm{KH_{2 }} \mathrm{PO}_{4}$, and $0.05 \mathrm{M}$ acetic acid with the $\mathrm{pH}$ adjusted to 4.4 with $1 \mathrm{M} \mathrm{NaOH}$.

\subsection{Optical Coherence Tomography System Specification and Configuration}

The OCT system used for this study was custom designed and built in-house at Queen Mary University of London. The instrument incorporated a fiber optic Mach-Zehnder ${ }^{58}$ interferometer design utilizing a super-luminescent light-emitting diode optical source (SLD1325, Thorlabs Ltd., Cambridge, UK), operating with a nominal central wavelength of $1310 \mathrm{~nm}$ and bandwidth of $\sim 100 \mathrm{~nm}$. The axial and transverse resolutions were nominally 7 and $9 \mu \mathrm{m}$ in air, respectively.

Interference fringes were detected by a high-speed spectrometer, comprising an InGaAs linear detector array (SU-LDH2,

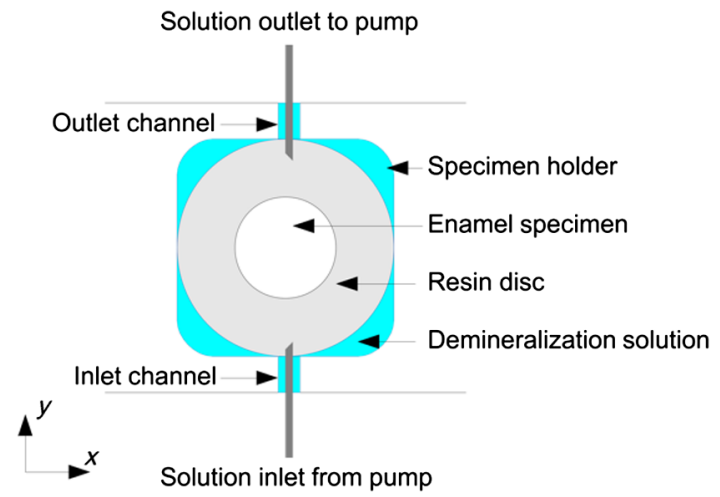

Fig. $1 X Y$-plane view of a bovine enamel specimen, mounted in a resin substrate and placed into the specimen holder. The holder and specimen were orientated vertically, immersed in demineralizing solution. The solution was continuously refreshed by a pump that introduced fresh solution through an inlet channel at the bottom of the specimen holder and removed solution through an outlet channel at the top.

Sensors Unlimited), a reflective diffraction grating and focusing/ collimating achromatic doublet lenses. Images were obtained by raster scanning the probe beam over the tissue surface using a two-dimensional galvanometer configuration (GVS012, Thorlabs, Cambridge, UK). The light was focused beneath the sample surface by a scan lens (Thorlabs LSM03). The system acquired and processed 3-D OCT data in real time using a general-purpose graphics processing unit (NVIDIA C2070). ${ }^{20}$

A multispecimen holder was fabricated from transparent poly(methyl methacrylate) (PMMA) comprising five isolated flow cell chambers. A typical chamber is shown in Fig. 1, each chamber measured $24 \times 24 \times 4 \mathrm{~mm}$ (width $\times$ height $\times$ depth), having an actual volume of $2.3 \mathrm{~mL}$. The true volume was slightly less than the cuboid volume due to rounded chamber edges. A single piece PMMA lid covered the compartments with each being isolated by a rubber O-ring. The chambers accommodated $\sim 0.8 \mathrm{~mL}$ of solution with the specimen and resin disk occupying the remaining volume $(1.5 \mathrm{~mL})$. An inlet channel was placed at the bottom of each chamber with an outlet placed at the top. Solution was flowed through the chambers at a rate of $5 \mathrm{~mL} / \mathrm{min}$ by a motorized pump (323 Du/D, Watson Marlow, UK). Solution was coupled in and out of the chambers by 21 gauge syringes.

The OCT probe was mounted onto a linear translation stage (LTS300/M, Thorlabs, UK) such that the probe beam propagated horizontally, parallel to the lab bench. The specimens were mounted vertically in the specimen holder with the exposed enamel face perpendicular to the OCT probe beam. This configuration is shown in Fig. 2.

\subsection{Calibration}

The axial collection efficiency of the OCT system and the sensitivity roll-off were calibrated by measuring a weakly scattering phantom. ${ }^{23}$ This has been previously described as a point spread function phantom, ${ }^{59,60}$ comprising a low concentration of $\mathrm{FeO}$ nanoparticles having an average interparticle spacing of $50 \mu \mathrm{m}$. The well-spaced particles yielded $\mu_{\text {cal }} \ll \mu_{\mathrm{OCT}}$ and thus the effect of signal attenuation due to scattering was considered negligible. An area of the phantom surface measuring $3 \times 3 \mathrm{~mm}$ was measured using the OCT system. The phantom was mounted in the specimen holder and submerged in deionized 


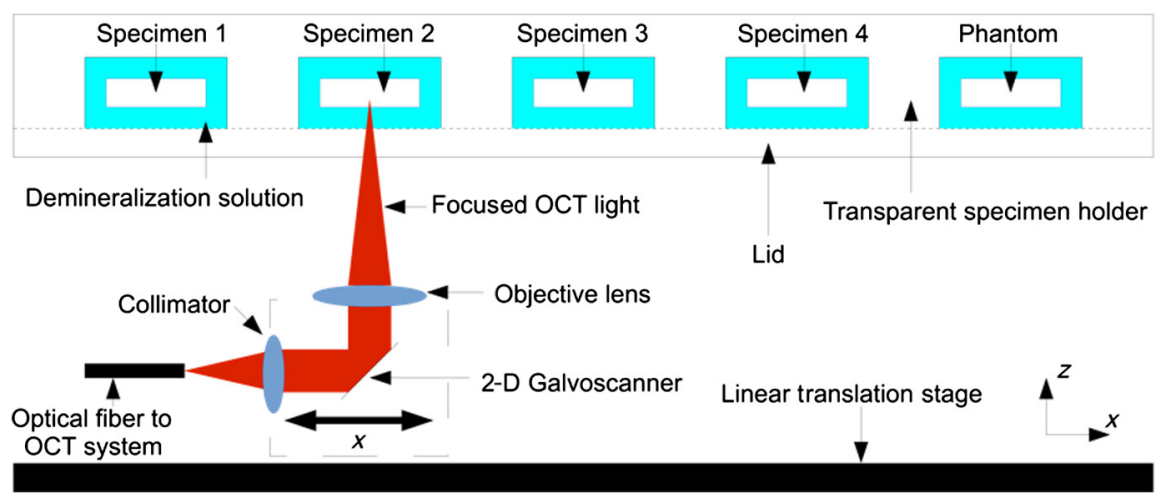

Fig. $2 X Z$-plane view of the experimental configuration. Four bovine enamel specimens and one scattering phantom were mounted vertically in a transparent specimen holder, through which the demineralizing solution was continuously flowed. 3-D OCT volumes of each were obtained automatically by moving the OCT probe between specimens on a motorized linear translation stage.

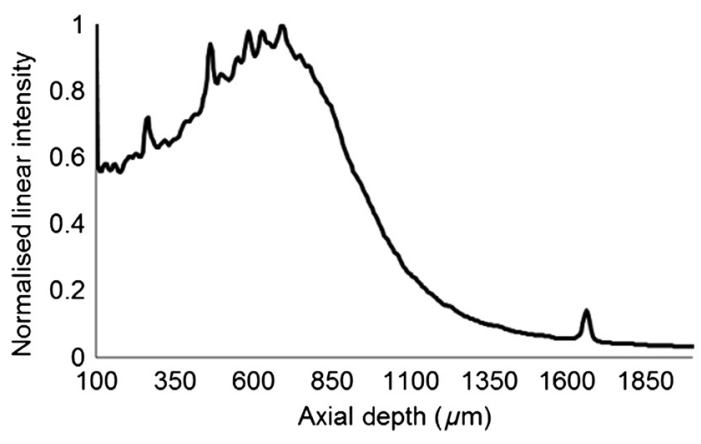

Fig. 3 The calibration A-scan for the OCT system used in this study. Several small sharp peaks are visible, which are due to the nonuniform system noise floor.

water to the match the configuration of the demineralization experiments. The mean of 250,000 A-scans was used to represent the average calibration curve by which all subsequent OCT A-scans of the specimens were divided. The calibration curve is shown in Fig. 3. The curve is interrupted by several small sharp peaks that correspond with the spatial frequencies of spurious spectrometer signals and background OCT system reflections.

\subsection{Measurement Protocol}

The specimens were initially stored dry in sealed containers at a temperature of $23^{\circ} \mathrm{C}$. The samples were immersed in deionized water for $24 \mathrm{~h}$ prior to initiation of demineralization and OCT measurement. This mitigated against hydration effects influencing the OCT signal during the initial stages of the experiment.

Four hydrated specimens and one reference phantom were placed into each well of the specimen holder. The reference phantom was a polished epoxy block, loaded with $\mathrm{TiO}_{2}$ scattering particles having a known scattering coefficient ${ }^{23}$ of $\mu_{\mathrm{s}}=$ $3.5 \mathrm{~mm}^{-1}$, thus providing a constant benchmark for the OCT measured light backscattering intensity. The polished enamel surface was orientated toward the OCT probe beam as shown in Fig. 2. Initially, deionized water was pumped into the flow cell and the central region of the specimen imaged using the OCT system. This was accomplished under computer control such that the alignment of the experimental system was not disturbed. The water was then removed from the specimen wells and replaced with demineralization solution in each chamber using the computer-controlled pump. Filling and emptying of the chambers were completed in a few seconds at a flow rate of $5 \mathrm{~mL} / \mathrm{min}$. However, during the acid challenge, the solution was flowed continuously at a rate of $0.1 \mathrm{~mL} / \mathrm{min}$. The solution was not recycled but drawn from a reservoir of stock demineralization solution. The OCT system automatically acquired a 3-D dataset from a set location within each sample at a minimum of 1-h intervals. Therefore, longitudinally acquired OCT image volumes of each specimen were spatially coregistered.

\subsection{Lesion Depth and Optical Properties Measurement}

Quantitative analysis of the OCT measurements was automated from the raw data using a script written in the MATLAB ${ }^{\circledR}$ programming language. OCT A-scans were processed from the detected spectral interference patterns into linear intensity profiles. The approximate specimen surface location was known $a$ priori due to the nature of the experimental setup and specimen holder. This information was used to approximately locate the surface reflection peak. The mean of 100 adjacent A-scans was found, from which the intensity maximum was assumed to represent the specimen surface location.

The lesion depth, $d_{\text {lesion }}$, was estimated by fitting the sum of two Gaussian functions of the form given in the below equation to the OCT signal intensity region around the lesion, $I_{\text {lesion }}$

$I_{\text {lesion }}=A_{1} \exp \left[-\frac{\left(z-z_{1}\right)^{2}}{w_{1}^{2}}\right]+A_{2} \exp \left[-\frac{\left(z-z_{2}\right)^{2}}{w_{2}^{2}}\right]$.

In the above equation, the two Gaussian functions are denoted by the subscript $n=1$ and 2 . The peak height of the functions is given by $A$, peak location $z_{n}$, and $1 / e$ half width $w$. Thus, the lesion depth was estimated as

$d_{\text {lesion }}=z_{2}-z_{1}+\frac{1}{2}\left(w_{2}+w_{1}\right)$.

Subtracting a systematic offset from the final value to ensure that the lesion thickness was always zero at $t=0$.

This method was chosen empirically because it proved to be stable for automation, comparing well with manual measurements. Specifically, $w_{1}$ and $w_{2}$ were incorporated into Eq. (7) 


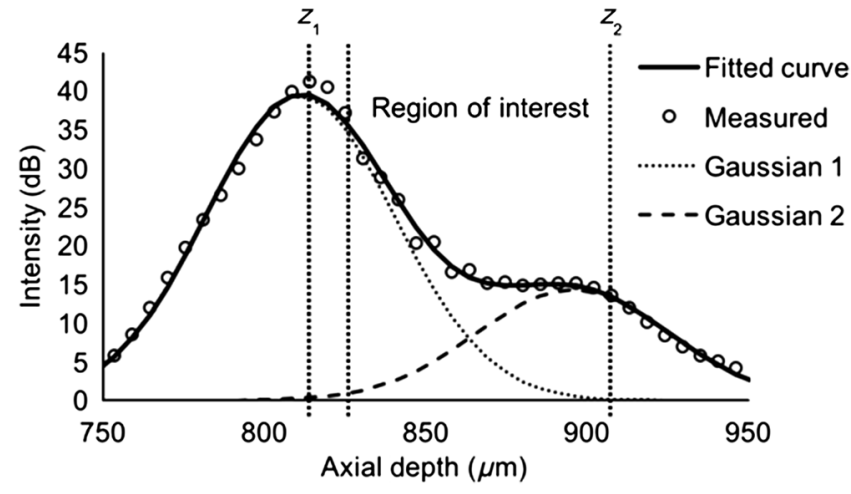

Fig. 4 Section of a mean A-scan for a demineralization lesion. The circles show the measured data points compared to the fitted sum of Gaussians (solid line). The dotted and dashed lines show the two individual Gaussian functions.

because they increased they sensitivity of $d_{\text {lesion }}$ both to the varying reflection caused by surface erosion and demineralization at the lesion front as it extended into the sound enamel. A typical fit of Eq. (6) to a measured lesion mean A-scan is shown in Fig. 4 as a solid line compared to the measured data points (circles). The two Gaussian functions are shown independently as dotted and dashed lines.

Parameters obtained from the lesion depth fit were also used to define a region of interest (ROI) from which the optical properties were estimated. Typically, the peak reflection was high compared to the backscattered intensity from beneath the surface due to the refractive index mismatch between the demineralizing solution and the tooth. Therefore, the peak was filtered out by selecting an ROI from the A-scan, starting 2 pixels $(\approx 10 \mu \mathrm{m})$ below the surface peak at $z_{2}$ and extending across the lesion to $z_{2}$. The ROI selection is indicated in Fig. 4 .

Equation (3) was fitted to the extracted subregion, providing output estimates of $\mu_{\mathrm{OCT}}^{\prime}(t)$ and $\mu_{\mathrm{B}}^{\prime}(t)$. For each series of longitudinal measurements, the percentage change in $\mu_{\mathrm{B}}^{\prime}(t)$ was calculated at each measurement time $t$ as

$$
\Delta \mu_{\mathrm{B}}^{\prime}(t)=100 \% \frac{\Delta \mu_{\mathrm{B}}^{\prime}(t)-\Delta \mu_{\mathrm{B}}^{\prime}(0)}{\Delta \mu_{\mathrm{B}}^{\prime}(0)} .
$$

Assuming $\mu_{\mathrm{cal}} \ll \mu_{\mathrm{OCT}}$ and $a \approx 1$, then $\mu_{\mathrm{OCT}}^{\prime} \approx \mu_{\mathrm{s}}$. Therefore, from here on, the measured attenuation coefficient is referred to as the scattering coefficient. The mean scattering coefficient and the corresponding standard error of the mean were calculated at each time point across all specimens within the model group.

\section{Results}

Central B-scans from the citric, acetic, and caries-like models are shown in Figs. 5, 6, and 7 respectively, at baseline (a) and following acid challenge of $6 \mathrm{~h} \mathrm{(b),} 12 \mathrm{~h}$ (c), and $24 \mathrm{~h}$ (d). Visually, all three models followed similar patterns of progression. At baseline, the dentine-enamel junction was visible, but this became increasingly difficult to discern with increasing duration of acid challenge. At and below the surface, a distinct band of high backscatter (lighter region) was observed to form. Following $6 \mathrm{~h}$ of continuous acid challenge, the citric acid model [Fig. 5(b)] showed signs of surface roughening, departing from the smooth profile in Fig. 5(a). Following $24 \mathrm{~h}$ of acid challenge, there was visible erosion of the enamel surface relative to the varnished region.

The acetic acid model showed less axial erosion. However, the band of intense backscatter extended deeper beneath the surface following $24 \mathrm{~h}$ of acid challenge compared with the citric acid model [see Figs. 5(d) and 6(d)].

The caries-like model in Fig. 7 similarly developed a band of intense backscatter, increasing in subsurface depth with acid exposure time. The band itself extended to a similar depth to the citric acid model following $24 \mathrm{~h}$ of acid challenge [Fig. 7(d)]. However, there was no perceivable erosion or surface change.

The experimental setup ensured spatio-temporal registration of the OCT images. This facilitated direct comparison of quantitative parameters measured from the OCT data. The subsurface lesion depth was measured for each specimen. The mean subsurface lesion depths were compared for each model by plotting their change throughout the acid challenge, Figs. 8(a)-8(c), where the error bars represent the standard error of the mean calculated across the four specimens in each group. The citric acid model [Fig. 8(a)] yielded a maximum subsurface lesion depth of $76 \pm 4 \mu \mathrm{m}$ compared with $177 \pm 8 \mu \mathrm{m}$ and $100 \pm$ $4 \mu \mathrm{m}$ for the acetic [Fig. 8(b)] and caries-like [Fig. 8(c)] models, respectively. The citric and acetic acid models exhibited a twostage behavior in the lesion depth formation. This can be seen in Fig. 8(a) (citric) at $t=10 \mathrm{~h}$ and Fig. 8(b) (acetic) at $t=24 \mathrm{~h}$.

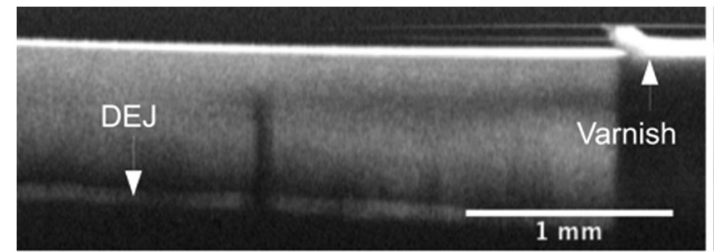

(a)

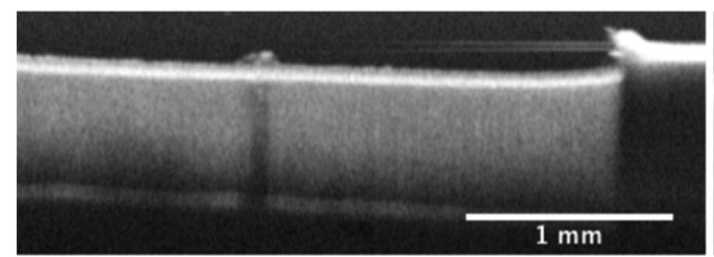

(c)

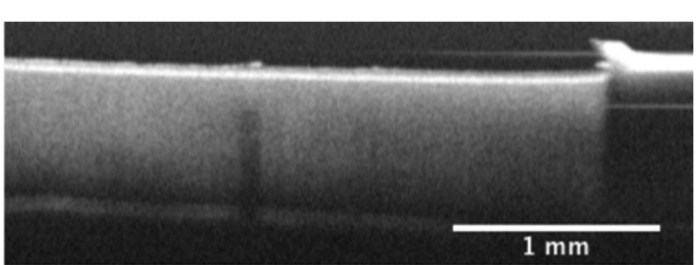

(b)

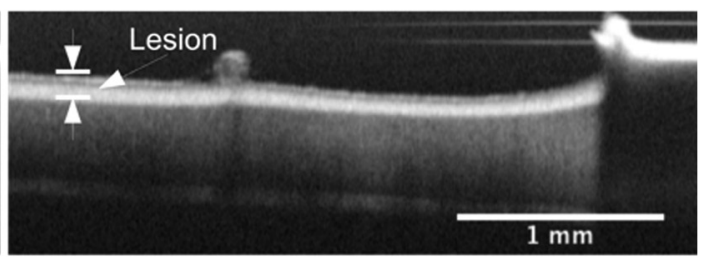

(d)

Fig. 5 Registered OCT B-scans of bovine enamel, (a) hydrated prior to acid challenge and following (b) $t=6 \mathrm{~h}$, (c) $t=12 \mathrm{~h}$, and (d) $t=24 \mathrm{~h}$ of continuous exposure to a citric acid solution at $\mathrm{pH} 3.8$. 


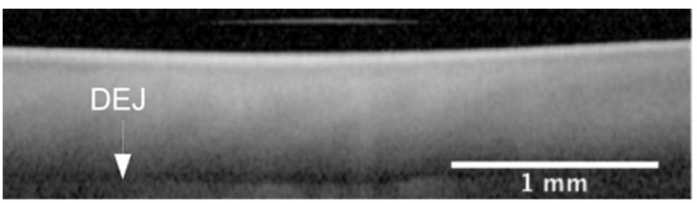

(a)

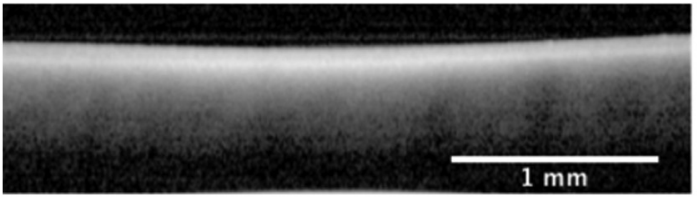

(c)

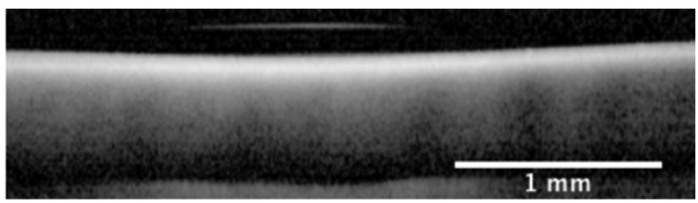

(b)

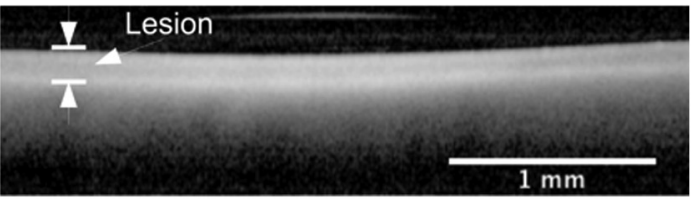

(d)

Fig. 6 Registered OCT B-scans of bovine enamel, (a) hydrated prior to acid challenge and following (b) $t=6 \mathrm{~h}$, (c) $t=12 \mathrm{~h}$, and (d) $t=24 \mathrm{~h}$ of continuous exposure to an acetic acid solution at $\mathrm{pH} 4.0$.

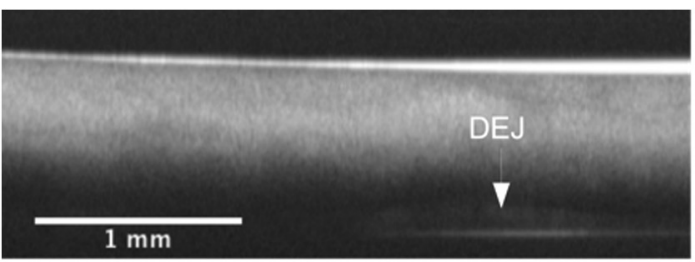

(a)

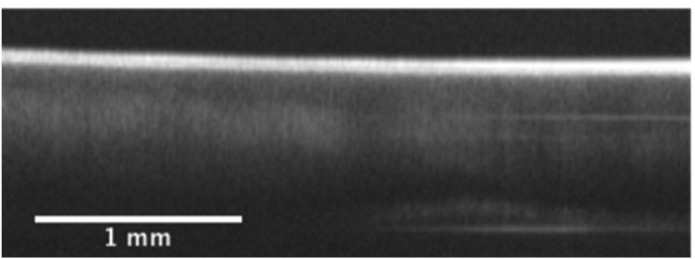

(c)

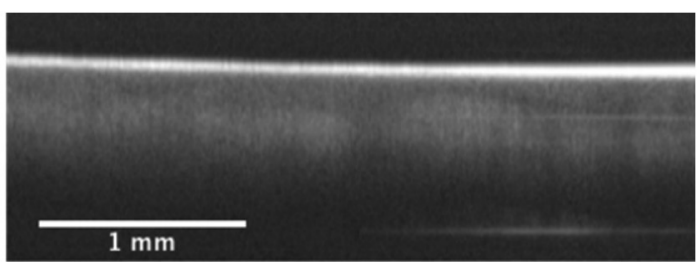

(b)

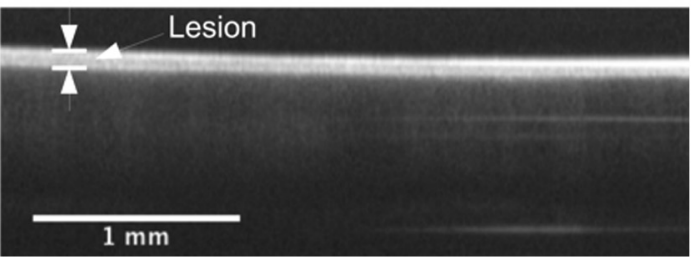

(d)

Fig. 7 Registered OCT B-scans of bovine enamel, (a) hydrated prior to acid challenge and following (b) $t=6 \mathrm{~h}$, (c) $t=12 \mathrm{~h}$, and (d) $t=24 \mathrm{~h}$ of continuous exposure to an acetic acid/calcium phosphate solution at $\mathrm{pH} 4.4$.

The lesion depth plateaued followed by an instantaneous increase in the growth rate. A similar phenomenon is not clearly evident in Fig. 8(c) for the caries-like model, for which the lesion depth growth rate appeared to be linear for $t>6 \mathrm{~h}$. However, there is arguably a subtle increase in the lesion growth rate around $36 \mathrm{~h}$.
The citric and acetic acid solutions are necessarily erosive, albeit with a different $\mathrm{pH}$ and ionic composition. Thus, Fig. 8 contains only part of the information with regard to lesion depth. The total lesion depth includes the depth of enamel lost from the surface, shown visually in Fig. 5(d). The sum of surface enamel loss and the subsurface lesion depth is shown in Fig. 9

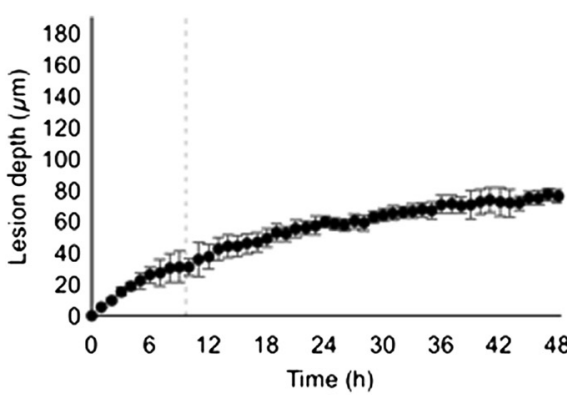

(a)

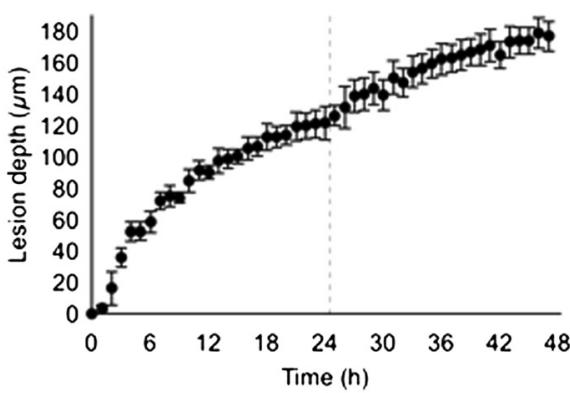

(b)

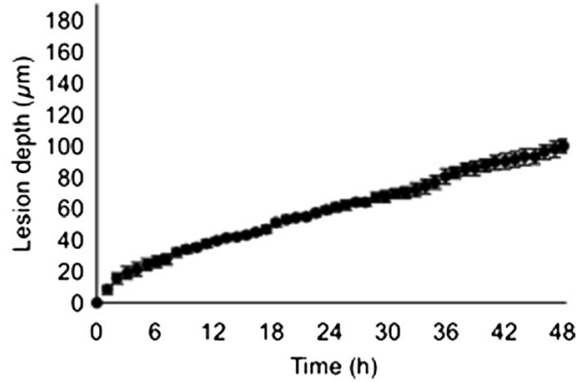

(c)

Fig. 8 Subsurface lesion depth measured from mean OCT B-scans for three different models. (a) Citric acid solution at pH 3.8, (b) acetic acid solution at $\mathrm{pH} 4.0$, and (c) acetic acid solution containing calcium chloride and potassium phosphate buffered to $\mathrm{pH}$ 4.4. The vertical line indicates a discontinuous change in the lesion depth gradient. 


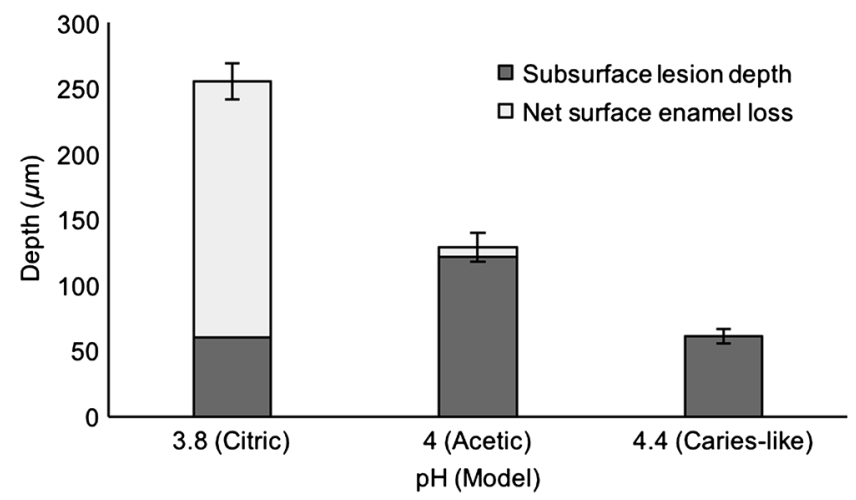

Fig. 9 Total lesion depth after $24 \mathrm{~h}$ of continuous acid challenge, representing the sum of the subsurface lesion depth (dark gray) and the depth of surface enamel loss (light gray). The error bar represents the standard error of the mean for total lesion depth.

for all three models at $t=24 \mathrm{~h}$, with error bars representing the standard deviation of the total.

The citric acid and caries-like models showed statistically similar subsurface lesion depth measurements ( $t$-test, $p>0.05$ ). However, the citric model was dominated by surface erosion that was absent from the latter due to the addition of calcium chloride and potassium phosphate to the demineralizing solution, which served to maintain the enamel surface. The acetic acid model produced a deeper subsurface lesion, with a relatively small amount of surface erosion. These different lesion characteristics also manifested in terms of the lesion scattering properties, shown in Figs. 10(a)-10(c). The peak scattering coefficient values are in the range $24 \pm 2$ to $35 \pm 3 \mathrm{~mm}^{-1}$, consistent with values obtained by light transmission measurements. ${ }^{61}$ However, attenuation values were observed to initially increase to these maxima, followed by a decrease to approximately between 0 and $5 \mathrm{~mm}^{-1}$. The acetic acid model [Fig. 10(b)] shows a drop in the attenuation coefficient to $-7 \pm 3 \mathrm{~mm}^{-1}$, indicating an axially increasing OCT signal intensity.

Nevertheless, the postmaximum attenuation curves are visually similar between the models, with a few distinct differences. Each of the models produced attenuation curves with different vertical offsets, i.e., citric acid resulted in a range of attenuation values from $2 \pm 2$ to $27 \pm 4 \mathrm{~mm}^{-1}$, acetic acid in a range of $-7 \pm 3$ to $24 \pm 2$, and the caries-like model in a range of $5 \pm$ 5 to $38 \pm 1$. Similarly, the curves for the different models appear to also be offset in time. Figure 10(a) appears to be shifted left with respect to Figs. 10(b) and 10(c) shifted right by $\sim 6 \mathrm{~h}$. The different models also appear to be scaled differently along the time axis.

The backscattering coefficient, however, exhibited distinctly different behavior between the citric model and both acetic acidbased models. The citric acid model [Fig. 11(a)] yielded a continuous backscattering coefficient decrease of $61 \% \pm 5 \%$ at 48 h. However, both the acetic [Fig. 11(b)] and caries-like [Fig. 11(c)] models show an initial increase $(83 \% \pm 40 \%$ and $248 \% \pm 65 \%$, respectively) in the backscattering coefficient followed by a decrease. Notably, after $48 \mathrm{~h}$ of acid challenge, the acetic and caries-like models result in a net backscattering coefficient increase of $31 \% \pm 16 \%$ and $139 \% \pm 34 \%$, respectively.

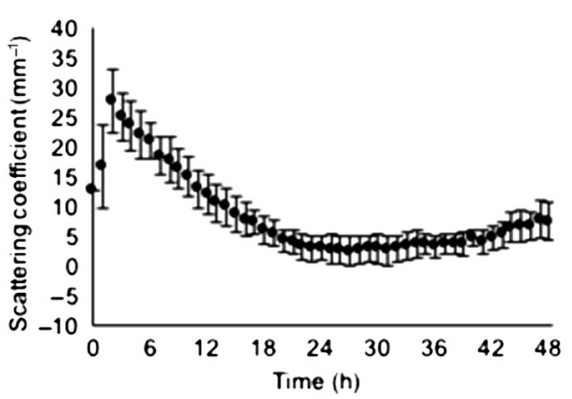

(a)

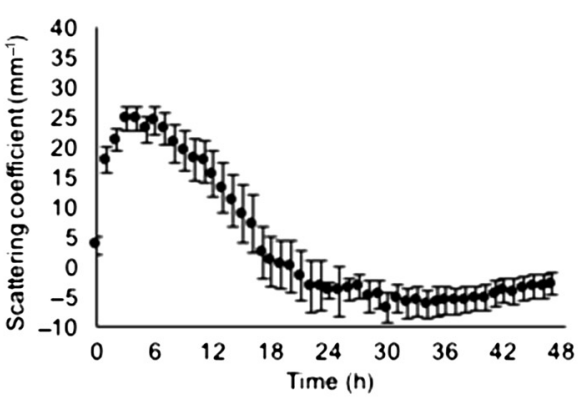

(b)

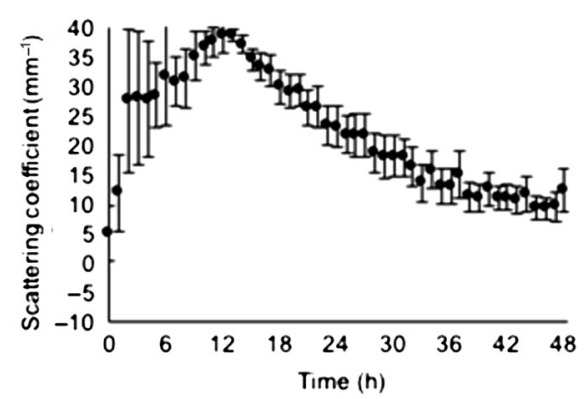

(c)

Fig. 10 Scattering coefficient, estimated from mean OCT A-scans for three different models. (a) Citric acid solution at $\mathrm{pH} 3.8$, (b) acetic acid solution at $\mathrm{pH} 4.0$, and (c) acetic acid solution containing calcium chloride and potassium phosphate buffered to $\mathrm{pH} 4.4$.

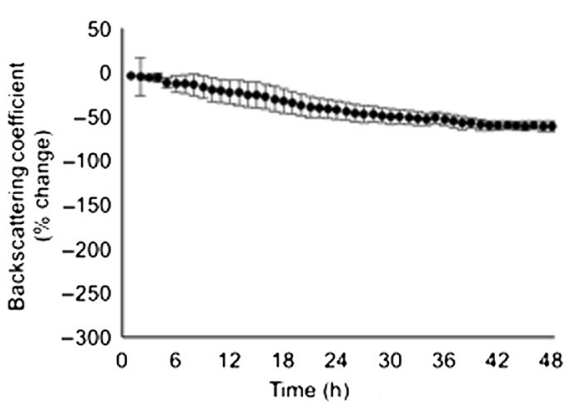

(a)

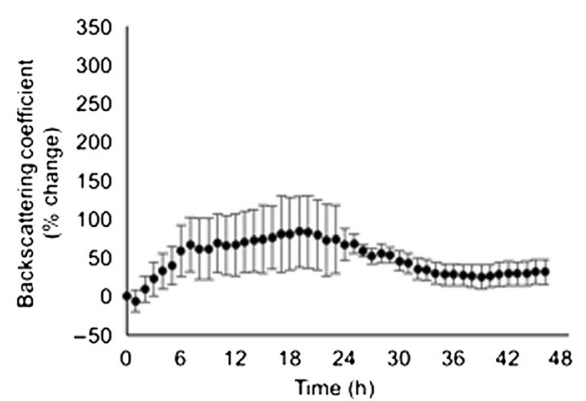

(b)

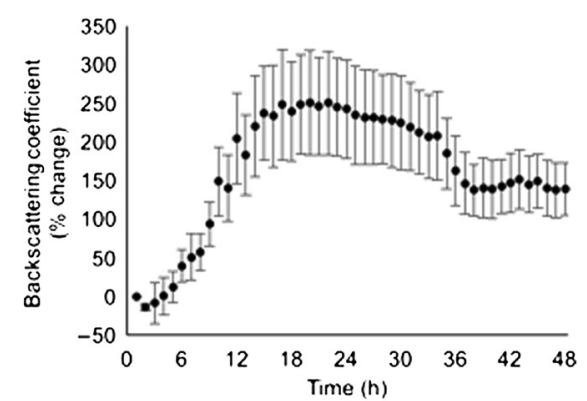

(c)

Fig. 11 Percentage change in the backscattering coefficient $\left(\mu_{\mathrm{B}}\right)$, estimated from mean OCT A-scans for three different models. (a) Citric acid solution at $\mathrm{pH} 3.8$, (b) acetic acid solution at $\mathrm{pH} 4.0$, and (c) acetic acid solution containing calcium chloride and potassium phosphate buffered to $\mathrm{pH} 4.4$. 


\section{Discussion}

This work has investigated the temporal dynamics of three bovine enamel demineralization models using an automated four-dimensional OCT system integrated with a multispecimen flow cell. This configuration maintained the spatial registration of the OCT image volumes facilitating analysis on the same tissue regions at each acquisition. The specimens were not handled or moved throughout the experiment, thus removing this previously reported source of uncertainty.

Figures 8, 10, and 11 all demonstrate that lesion properties vary with time and support the finding that the different models produce detectable differences within the OCT data. The subsurface lesion depth dynamics are distinct between all models. The citric and caries-like lesions showed statistically similar lesion depths at $24 \mathrm{~h}$. However, when plotted with time (Fig. 8), the lesion progression followed a visually different shape. All models produced a subsurface lesion. However, in the citric and acetic models, the depth to which this extended plateaued. This is consistent with the net removal of surface enamel (Fig. 9), which limited the depth of subsurface lesion development. Similar rate limiting behavior has previously been observed in a microradiographic study. ${ }^{62}$ Furthermore, the same study also reported a secondary increase in the demineralization rate at $24 \mathrm{~h}$ for a similar $\mathrm{pH} 4.0$ acetic acid model to that used in this work. This coincides with the discontinuous rate of lesion depth formation observed by OCT in Figs. 8(a) and 8(b), although the exact reason for this behavior is yet to be elucidated. In their work, Anderson et al. attributed it to formation of a surface layer overlying subsurface demineralization. The OCT backscattering coefficient measurements in Fig. 11(b) lend support to this hypothesis with the backscattering maximum observed shortly before the change in lesion depth rate. A similar change is evident in Fig. 11(c), which could support a lesion rate change close to $36 \mathrm{~h}$. There is no corresponding backscattering change in Fig. 11(a), possibly due to the rapid formation of a surface layer. $^{63}$

Ignoring differences in ionic composition, the lesion depth results indicate that increasing the $\mathrm{pH}$ allowed formation of a deeper subsurface lesion while minimizing the loss of surface enamel. It was expected ${ }^{63}$ that the addition of calcium and phosphate to the demineralization solution resulted in more carieslike lesion formation, maintaining an intact surface. Figure 7 appears to support this, showing little or no change to the surface reflection. However, in this work, the caries-like forming solution has the highest $\mathrm{pH}$ at 4.4. The $\mathrm{pH}$ scale is logarithmic with respect to hydrogen ion concentration, resulting in a $60 \%$ reduction in the caries-like solution compared to the acetic model. Therefore, the results, in this paper, cannot dismiss the possibility that the appearance of an intact surface is a consequence of a slower dissolution of surface enamel. Nevertheless, no surface changes were detected in the OCT data.

Measurements of $\mu_{\mathrm{OCT}}$ produced discernible differences between each lesion type (Fig. 10). Visually, there was a time offset between the three models, supported by the dissolution rate dependence upon $\mathrm{pH}^{64}$ However, the theory on which OCT signal slope measurement is based assumes a uniform scattering material. Unfortunately, partial demineralization necessarily leads to local inhomogeneity. Thus, caution must be exercised when quantitatively interpreting these results. The calibration step provides a normalization of the results for reproducibility. However, absolute values of $\mu_{\mathrm{OCT}}$ remain to be validated. Nevertheless, the formation of demineralized lesions does alter the local OCT signal intensity and slope (Figs. 5-7). This study attempted to capture this local variation by fitting Eq. (1) to an ROI within the lesion body. The B-scan images [Figs. 5(d), 6(d), and 7(d)] show an increase in backscattered signal intensity at the leading edge of the lesion. Assuming the relationship $\mu_{\mathrm{B}} / \mu_{\mathrm{s}}=p_{\mathrm{NA}}$ holds (see Ref. 26), then this might be interpreted as a discontinuous increase in $\mu_{\mathrm{s}}$ with a consequent local increase in $\mu_{\mathrm{B}}$. Somewhat counter-intuitively, this resulted in negative measurements of $\mu_{\mathrm{OCT}}$. This is not physical in the sense that the material provides no gain. However, it does reflect the OCT signal behavior as its intensity increases from the lesion body to its leading edge. One interpretation of this is that initial demineralization removes a small amount of mineral, causing a large refractive index discontinuity between enamel prisms resulting in a large, localized increase in scattering. Continued demineralization decreases the enamel concentration and, therefore, scattering decreases as the lesion body develops. These dynamics were captured in this study by fitting a curve to the lesion and, in this way, demonstrated sensitivity to relative changes with respect to time and demineralization model. However, physical interpretation of the measured values of $\mu_{\mathrm{OCT}}$, including the impact of multiple scattering, remains open for discussion.

Nevertheless, the results in this paper are of substantial importance. They demonstrate that OCT can be used to detect the different optical signatures associated with different demineralization models and track their dynamics. Further study of alternative models with different physiological significance could provide baseline measurements to which clinical OCT data could be compared and physically interpreted.

\section{Disclosures}

No conflicts of interest, financial or otherwise, are declared by the authors.

\section{Acknowledgments}

Abdi Aden was sponsored by GlaxoSmithKline (GSK) through the Biotechnology and Biological Sciences Research Council Industrial CASE award BB/K501190/1. The authors gratefully acknowledge Dr. Gary Burnett and Dr. Richard lynch from GSK for helpful discussion and providing the Bovine enamel specimens used in this work.

\section{References}

1. N. Garg and A. Garg, Textbook of Operative Dentistry, Jaypee Brothers Medical Publishers, New Delhi, India (2010).

2. A. I. Ismail et al., "The International Caries Detection and Assessment System (ICDAS): an integrated system for measuring dental caries," Community Dent. Oral Epidemiol. 35(3), 170-178 (2007).

3. R. S. Jones and D. Fried, "Remineralization of enamel caries can decrease optical reflectivity," J. Dent. Res. 85(9), 804-808 (2006).

4. G. H. Sperber and M. G. Buonocore, "Effect of different acids on character of demineralization of enamel surfaces," J. Dent. Res. 42(2), 707-723 (1963).

5. B. T. Amaechi, S. M. Higham, and W. M. Edgar, "Use of transverse microradiography to quantify mineral loss by erosion in bovine enamel," Caries Res. 32(5), 351-356 (1998).

6. J. Arends, J. Schuthof, and W. G. Jongebloed, "Lesion depth and microhardness indentations on artificial white spot lesions," Caries Res. 14(4), 190-195 (1980).

7. J. D. B. Featherstone et al., "Comparison of artificial caries-like lesions by quantitative microradiography and microhardness profiles," Caries Res. 17(5), 385-391 (1983). 
8. L. Holmen et al., "A scanning electron microscopic study of progressive stages of enamel caries in vivo," Caries Res. 19(4), 355-367 (1985).

9. G. R. Davis, A. N. Z. Evershed, and D. Mills, "Quantitative high contrast x-ray microtomography for dental research," J. Dent. 41(5), 475-482 (2013).

10. G. R. Davis and F. S. L. Wong, "X-ray microtomography of bones and teeth," Physiol. Meas. 17(3), 121-146 (1996).

11. A. M. A. Maia et al., "Evaluation of dental enamel caries assessment using quantitative light induced fluorescence and optical coherence tomography: enamel caries detection: QLF and OCT," J. Biophotonics 9(6), 596-602 (2016).

12. H. Kang et al., "Nondestructive assessment of early tooth demineralization using cross-polarization optical coherence tomography," IEEE J. Sel. Top. Quantum Electron. 16(4), 870-876 (2010).

13. B. T. Amaechi et al., "Use of optical coherence tomography for assessment of dental caries: quantitative procedure," J. Oral Rehabil. 28(12), 1092-1093 (2001).

14. Y. Shimada et al., "Noninvasive cross-sectional imaging of proximal caries using swept-source optical coherence tomography (SS-OCT) in vivo," J. Biophotonics 7(7), 506-513 (2014).

15. A. Baumgartner et al., "Polarization-sensitive optical coherence tomography of dental structures," Caries Res. 34(1), 59-69 (2000).

16. D. Fried et al., "Imaging caries lesions and lesion progression with polarization sensitive optical coherence tomography," J. Biomed. Opt. 7(4), 618-627 (2002).

17. H. P. Chew et al., "Measuring initial enamel erosion with quantitative light-induced fluorescence and optical coherence tomography: an in vitro validation study," Caries Res. 48(3), 254-262 (2014).

18. W. F. Cheong, S. A. Prahl, and A. J. Welch, "A review of the optical properties of biological tissues," IEEE J. Quantum Electron. 26(12), 2166-2185 (1990).

19. E. S. Papazoglou et al., "Changes in optical properties of tissue during acute wound healing in an animal model," J. Biomed. Opt. 13(4), 044005 (2008).

20. L. Karlsson, "Caries detection methods based on changes in optical properties between healthy and carious tissue," Int. J. Dent. 2010, 1-9 (2010).

21. S. Anand et al., "Estimation of tissue optical properties between different grades and stages of urothelial carcinoma using diffuse reflectance spectroscopy," in Int. Conf. on BioPhotonics, pp. 1-3 (2015).

22. S. L. Jacques, "Optical properties of biological tissues: a review," Phys. Med. Biol. 58(11), R37 (2013).

23. M. Almasian et al., "Validation of quantitative attenuation and backscattering coefficient measurements by optical coherence tomography in the concentration-dependent and multiple scattering regime," J. Biomed. Opt. 20(12), 121314 (2015).

24. D. Faber et al., "Quantitative measurement of attenuation coefficients of weakly scattering media using optical coherence tomography," Opt. Express 12(19), 4353-4365 (2004).

25. D. Levitz et al., "Determination of optical scattering properties of highly-scattering media in optical coherence tomography images," Opt. Express 12(2), 249-259 (2004).

26. V. M. Kodach et al., "Determination of the scattering anisotropy with optical coherence tomography," Opt. Express 19(7), 6131-6140 (2011).

27. N. Choudhury and S. L. Jacques, "Extracting scattering coefficient and anisotropy factor of tissue using optical coherence tomography," Proc. SPIE 8221, 822111 (2012).

28. S. Prahl, "Light transport in tissue," PhD Thesis, University of Texas Austin (1988)

29. B. Povazay et al., "Three-dimensional optical coherence tomography at $1050 \mathrm{~nm}$ versus $800 \mathrm{~nm}$ in retinal pathologies: enhanced performance and choroidal penetration in cataract patients," J. Biomed. Opt. 12(4), 041211 (2007).

30. V. M. Kodach et al., "Quantitative comparison of the OCT imaging depth at $1300 \mathrm{~nm}$ and $1600 \mathrm{~nm}$," Biomed. Opt. Express 1(1), 176-185 (2010).

31. J. C. Elliott et al., "Determination of mineral concentration in dental enamel from x-ray attenuation measurements," Connect. Tissue Res. 38(1-4), 61-72, Discussion 73-79 (1998).

32. Y. Shimamura et al., "Influence of tooth-surface hydration conditions on optical coherence-tomography imaging," J. Dent. 39(8), 572-577 (2011).
33. C. L. Darling, G. D. Huynh, and D. Fried, "Light scattering properties of natural and artificially demineralized dental enamel at $1310 \mathrm{~nm}$," J. Biomed. Opt. 11(3), 034023 (2006).

34. D. Fried et al., "Nature of light scattering in dental enamel and dentin at visible and near-infrared wavelengths," Appl. Opt. 34(7), 1278-1285 (1995).

35. D. Levitz et al., "Quantitative characterization of developing collagen gels using optical coherence tomography," J. Biomed. Opt. 15(2), 026019 (2010).

36. J. M. Schmitt, A. Knüttel, and R. F. Bonner, "Measurement of optical properties of biological tissues by low-coherence reflectometry," Appl. Opt. 32(30), 6032-6042 (1993).

37. F. J. van der Meer et al., "Localized measurement of optical attenuation coefficients of atherosclerotic plaque constituents by quantitative optical coherence tomography," IEEE Trans. Med. Imaging 24(10), 1369-1376 (2005).

38. G. van Soest et al., "Atherosclerotic tissue characterization in vivo by optical coherence tomography attenuation imaging," J. Biomed. Opt. 15(1), 011105 (2010).

39. O. K. Adegun et al., "Quantitative analysis of optical coherence tomography and histopathology images of normal and dysplastic oral mucosal tissues," Lasers Med. Sci. 27(4), 795-804 (2011).

40. J.-W. Su et al., "Precancerous esophageal epithelia are associated with significantly increased scattering coefficients," Biomed. Opt. Express 6(10), 3795-3805 (2015).

41. D. P. Popescu et al., "Assessment of early demineralization in teeth using the signal attenuation in optical coherence tomography images," J. Biomed. Opt. 13(5), 054053 (2008).

42. M. M. Mandurah et al., "Monitoring remineralization of enamel subsurface lesions by optical coherence tomography," J. Biomed. Opt. 18(4), 046006 (2013).

43. I. Hariri et al., "Effects of structural orientation of enamel and dentine on light attenuation and local refractive index: an optical coherence tomography study," J. Dent. 40(5), 387-396 (2012).

44. R. A. McLaughlin et al., "Parametric imaging of cancer with optical coherence tomography," J. Biomed. Opt. 15(4), 046029 (2010).

45. P. H. Tomlins et al., "Scattering attenuation microscopy of oral epithelial dysplasia," J. Biomed. Opt. 15, 066003 (2010).

46. A. Zvyagin et al., "Refractive index tomography of turbid media by bifocal optical coherence refractometry," Opt. Express 11, 3503-3517 (2003).

47. G. J. Tearney et al., "Determination of the refractive index of highly scattering human tissue by optical coherence tomography," Opt. Lett. 20(21), 2258-2260 (1995).

48. D. Segelstein, "The complex refractive index of water," MS Thesis, University of Missouri (1981).

49. Z. Meng et al., "Measurement of the refractive index of human teeth by optical coherence tomography," J. Biomed. Opt. 14(3), 034010 (2009).

50. M. E. Barbour et al., "Human enamel dissolution in citric acid as a function of $\mathrm{pH}$ in the range $2.30 \leq \mathrm{pH} \leq 6.30$ - a nanoindentation study," Eur. J. Oral Sci. 111(3), 258-262 (2003).

51. J. A. Hughes et al., "Effects of $\mathrm{pH}$ and concentration of citric, malic and lactic acids on enamel, in vitro," J. Dent. 28(2), 147-152 (2000).

52. A. Young and L. M. A. Tenuta, "Initial erosion models," Caries Res. 45(Suppl. 1), 33-42 (2011).

53. P. Anderson and J. C. Elliott, "Subsurface demineralization in dental enamel and other permeable solids during acid dissolution," J. Dent. Res. 71(8), 1473-1481 (1992).

54. K. H. Chan et al., "Methods for monitoring erosion using optical coherence tomography," Proc. SPIE 8566, 856606 (2013).

55. J. M. ten Cate et al., "Effect of timing of fluoride treatment on enamel de- and remineralization in vitro: a pH-cycling study," Caries Res. 22(1), 20-26 (1988).

56. J. J. M. Damen, M. J. Buijs, and J. M. ten Cate, "Fluoride-dependent formation of mineralized layers in bovine dentin during demineralization in vitro," Caries Res. 32(6), 435-440 (1998).

57. S. E. Jabbarifar et al., "Effect of fluoridated dentifrices on surface microhardness of the enamel of deciduous teeth," Dent. Res. J. 8(3), 113-117 (2011).

58. A. M. Rollins and J. A. Izatt, "Optimal interferometer designs for optical coherence tomography," Opt. Lett. 24, 1484-1486 (1999). 
59. P. D. Woolliams et al., "Spatially deconvolved optical coherence tomography," Appl. Opt. 49(11), 2014-2021 (2010).

60. P. D. Woolliams and P. H. Tomlins, "Estimating the resolution of a commercial optical coherence tomography system with limited spatial sampling," Meas. Sci. Technol. 22, 065502 (2011).

61. A. C. Chan et al., "Attenuation of near-IR light through dentin at wavelengths from 1300-1650-nm," Proc. SPIE 8929, 89290M (2014).

62. P. Anderson, M. Levinkind, and J. C. Elliott, "Scanning microradiographic studies of rates of in vitro demineralization in human and bovine dental enamel," Arch. Oral Biol. 43(8), 649-656 (1998).

63. H. C. Margolis et al., "Kinetics of enamel demineralization in vitro," J. Dent. Res. 78(7), 1326-1335 (1999).

64. H. M. Theuns et al., "Effect of the pH of buffer solutions on artificial carious lesion formation in human tooth enamel," Caries Res. 18(1), 7-11 (1984).

Biographies for the authors are not available. 